\title{
FATORES DE RISCO CARDIOVASCULAR EM ESCOLARES EGRESSOS DE UM PROGRAMA MULTIPROFISSIONAL DE EDUCAÇÃO EM SAÚDE
}

\section{CARDIOVASCULAR RISK FACTORS IN STUDENTS FROM A MULTIPROFESIONAL HEALTH EDUCATION PROGRAM}

\section{Lori da Conceição da França*, Helena Fraga-Maia**}

Autora para correspondência: Helena Fraga-Maia - helenafragamaia@gmail.com

*Fisioterapeuta da Clinica Santè

**Professora Adjunta na Universidade do Estado da Bahia

\section{R E S U M O}

Objetivo: Descrever a frequência de fatores de risco cardiovascular em escolares egressos de um programa multiprofissional de educação em saúde destinado a adoção de hábitos de vida saudável. Métodos: Realizou-se uma investigação com egressos do projeto "Doutores Mirins: Multiplicadores do Conhecimento". Foram convidados a participar escolares de 10 a 14 anos que tivessem obtido $75 \%$ de frequência no programa. Os dados foram analisados descritivamente. $O$ projeto foi aprovado pelo Comitê de Ética em Pesquisa da Universidade do Estado da Bahia (Parecer n. 241.434/2013) e financiado pelo PRO-Saúde/PET-Saúde 2012. Resultados: Observou-se que 65,8\% dos escolares eram do sexo feminino e $62,2 \%$ tinham idades variando de 12 a 13 anos. Dentre os fatores de risco cardiovascular observados encontravam-se a hipertensão arterial sistêmica (18,9\%), o sobrepeso $(32,4 \%)$, e o sedentarismo $(24,3 \%)$. O tempo de tela maior que duas horas foi reportado por $75,7 \%$ dos entrevistados. $O$ padrão alimentar descrito era, predominantemente, inadequado para $78,0 \%$ deles, com baixo consumo de saladas, frutas e verduras e elevada ingesta de alimentos ricos em açúcar, sódio e lipídios. Quanto aos pais, observou-se a escolaridade era baixa, assim como a renda familiar. Detectou-se ainda que grande parte dos genitores cursavam com Hipertensão Arterial Sistêmica (64,9\%). Conclusões: Os resultados permitem concluir que fatores ambientais e genéticos configuram um perfil de risco cardiovascular para os escolares justificando a necessidade de intervenções de educação em saúde mais duradouras e que envolvam a família, a escola e as unidades de atenção básica à saúde.

Palavras-Chave: Educação em Saúde; Escolares; Hipertensão Arterial Sistêmica; Fatores de risco cardiovascular. 
Objective: To describe the frequency of cardiovascular risk factors in schoolchildren from a multiprofesional health education program intended for the adoption of healthy lifestyles. Methods: An investigation was performed with students from the project: "Young Doctors - Multipliers of knowledge". Schoolchildren of ages from 10 to 14, who had obtained a $75 \%$ frequency in the program, were invited. The data was analyzed descriptively. The project was approved by the Ethics Committee in Research of Universidade do Estado da Bahia (Report 241.434/2013) and financed by PRO-Saúde/PET-Saúde 2012. Results: It was observed that $65.8 \%$ of the schoolchildren were of the female gender and $62.2 \%$ of ages varying between 12 and 13 years. Among the cardiovascular risk factors observed were systemic arterial hypertension (18.9\%), overweight $(32.4 \%)$, and sedentary lifestyles $(24.3 \%)$. Screen time greater than two hours was reported by $75.7 \%$ of the interviewed parties. The dietary pattern described was, predominantly, inadequate for $78.0 \%$ of the students, with low consumption of salad, fruit and vegetables and a high intake of food rich in sugar, sodium and lipids. In relation to the parents, it was observed that their schooling level was low, as well as their family income. It was also noted that a large share of the parents had Systemic Arterial Hypertension (64.9\%). Conclusions: The results permit us to conclude that environmental and genetic factors constitute a cardiovascular risk factor for the schoolchildren, justifying the necessity of longer-lasting interventions in health education and involving the families, the school and primary healthcare units.

Key words: Health education; Students; Systemic Arterial Hypertension; Cardiovascular risk factors. 


\section{INTRODUÇÃO}

As doenças crônicas não transmissíveis (DCNT) são, há muito, responsáveis por elevados índices de mortalidade e morbidade no mundo ${ }^{1,2}$. Elas são consideradas uma epidemia mundial pela Organização Mundial da Saúde (OMS), e responsáveis por aumento dos gastos em saúde'. Dentre estas, a Hipertensão Arterial Sistêmica (HAS) se destaca por ser a doença crônica de maior prevalência em todo $\circ$ mundo ${ }^{1,2}$. Trata-se de uma doença complexa e estudos genéticos e epidemiológicos têm sugerido que a hipertensão essencial é uma doença poligênica e multifatorial que resulta tanto de fatores genéticos quanto ambientais ${ }^{2}$.

Dentre os fatores ambientais que favorecem 0 aumento dos níveis pressóricos, sobressaem os relacionados ao estilo de vida, tais como hábitos alimentares inadequados, inatividade física, uso de álcool e tabagismo ${ }^{3-5}$. Muitos pesquisadores referem que estes hábitos encontram-se associados com a obesidade, um dos principais preditores da $\mathrm{HAS}^{3,4}$. Fatores genéticos têm sido, de modo semelhante, relacionados com a obesidade em crianças e adolescentes, e esta com a gênese das doenças cardiovasculares ${ }^{4}$.

Evidências científicas apontam que, em escolares, a coocorrência de hipertensão e excesso de peso, ou entre esta e outros fatores de risco cardiovascular tem se tornado mais frequente ${ }^{3}$, mas muitos deixam de receber o diagnóstico de hipertensão pela não realização de exames de rotina ${ }^{5}$. Deste modo, é possível que se deixe de diagnosticar precocemente e/ou intervir oportunamente em educação para uma vida saudável $l^{6}$. Nesse aspecto, a escola configurase como um local privilegiado para a aquisição de informações pouco disponíveis ${ }^{6}$. Estudos que abordaram essa temática, embora existentes, não enfocaram especificamente adolescentes que participaram de grupos de intervenção voltados para tais propósitos. Diante do exposto, o objetivo deste estudo foi descrever a frequência de fatores de risco cardiovascular em escolares egressos de um programa multiprofissional de educação em saúde, destinado a adoção de hábitos de vida saudável.
MATERIAIS E MÉTODOS

Realizou-se de um estudo transversal, descritivo, com escolares na faixa etária de 10 a 14 anos matriculados no Ensino Fundamental $2 \mathrm{em}$ uma escola da rede pública estadual no município de Salvador, Bahia, situada no Distrito Sanitário Cabula Beiru (DSCB). Adotou-se como critério de inclusão ter participado do programa de intervenção denominado de "Doutores Mirins: Multiplicadores do Conhecimento em HAS e Diabetes Mellitus" e obtido frequência superior a $75 \%$ dos encontros. Aqueles que aceitaram compor o grupo e que foram autorizados por seus pais a participarem da intervenção, foram os sujeitos da intervenção.

O referido projeto foi estabelecido em convênio com o Programa de Educação Pelo Trabalho para a Saúde - Pet Saúde/ Vigilância em Saúde Ambiental e Sanitária, financiado pelo Ministério da Saúde em parceria Secretaria Municipal da Saúde de Salvador e Universidade do Estado da Bahia. Tinha como objetivo idealizar e empregar estratégias metodológicas educativas e lúdicas em dez encontros semanais. Foram realizadas oficinas de conversas sobre as patologias, peças teatrais com fantoches e muitos jogos educativos. Ao final do projeto, 158 Doutores Mirins foram formados e, dentre estes, 46 cursavam o Ensino Fundamental 2 na rede pública estadual de ensino. Os demais cursavam o Ensino Fundamental 1 na rede municipal. Dados primários foram coletados pelos pesquisadores com a aplicação de um instrumento estruturado desenvolvido pelas autoras. A coleta de dados foi realizada, em média, após transcorrido cinco meses da finalização do projeto com o intuito de verificar o peso das contribuições dos ensinamentos no estilo de vida dos escolares. Deste modo, foram realizadas entrevistas de outubro de 2014 a abril de 2015.

Os escolares forneceram informações, tanto sobre a própria saúde, quanto sobre a saúde de seus pais. As variáveis mensuradas após o término do programa foram as medidas antropométricas, pressão arterial, assim como hábitos alimentares, história pregressa de saúde individual e familiar, e atividade física. Para aferição do peso corporal foi utilizada balança digital modelo W801 da marca Wiso $\AA$ com precisão de 100 gramas adotando-se 
a medida em quilogramas. Para tanto, o estudante deveria estar descalço e trajando roupas leves. A altura foi medida com estadiômetro $M D \circledR$, com precisão de 0,1 cm, e o Índice de Massa Corpórea (IMC) foi calculado por meio da fórmula (Peso/ Altura2) e classificado de acordo com os pontos de corte para crianças e adolescentes que variam de acordo com idade e sexo $0^{7}$. A pressão arterial foi aferida duas vezes com esfigmomanômetro aneroide adulto da marca Solidor ${ }^{\circledR}$ ou infantil da marca Premium ${ }^{\circledR}$, levando-se em conta a circunferência do braço e o peso da criança/adolescente, conforme preconizado pela VI Diretrizes Brasileiras de Hipertensão ${ }^{8}$. Para efeito de análise foi adotada a medida de maior valor. Foram utilizados os seguintes critérios: pressão arterial (PA) normal (PA < percentil 90); pré-hipertensão (PA entre percentis 90 a 95); HAS estágio 1 (percentil 95 a $99 \mathrm{mmHg}$ ); HAS estágio 2 (PA > percentil 99 mais $5 \mathrm{mmHg})^{8}$. Adotou-se sempre o critério de repouso por três minutos em que o escolar deveria manter-se sentado confortavelmente com os joelhos e o quadril flexionado a 90 graus e a bexiga vazia.

A cor da pele foi definida pelos entrevistadores e categorizada de acordo com o Instituto Brasileiro de Geografia e Estatística (IBGE) em branca, preta, parda, amarela e indígena. Os hábitos alimentares foram investigados de acordo com o Formulário de Marcadores do Consumo Alimentar normatizado pelo Protocolo do Sistema de Vigilância Alimentar e Nutricional (SISVAN) ${ }^{9}$ e desenvolvido para crianças com idade igual ou maior que cinco anos. Para tanto, aos escolares foram solicitadas informações sobre a frequência semanal do consumo de determinados alimentos nos últimos sete dias. $O$ nível de atividade física foi mensurado por um instrumento específico denominado de Questionário de Atividade Física para Adolescentes (QAFA) ${ }^{10}$, composto por uma lista com 24 atividades de intensidade moderada a vigorosa ( $\geq 3 M E T s$ ), e com possibilidade de o ado $\neg$ lescente acrescentar ainda mais duas outras opções. O tempo, mensurado em minutos semanais, era então computado. Foi adotado como ponto de corte 300 minutos, de modo que indivíduos acima desta margem foram considerados suficientemente ativos e abaixo da mesma classificados como insuficientemente ativos. $O$ tempo de tela foi definido como o tempo em frente à televisão, smartphone ou computador e categorizado em maior ou menor/ igual a 2 horas diárias, conforme ponto de corte definido também por outros autores ${ }^{10,11}$.

Informações sobre os pais foram requeridas para investigar os fatores ambientais e genéticos relacionados com os escolares. Assim, foram questionados sobre a ocupação destes, e esta foi categorizada em setor de serviços ou autônomos e desempregado(a). A escolaridade foi também investigada e definida em maior ou menor/igual a 8 anos. A renda familiar foi categorizada em maior ou menor/igual a 1 salário mínimo (SM). Com relação à saúde dos genitores foram realizadas questões sobre a presença de HAS, e se eles consideravam que seus pais cuidaram adequadamente dessa doença. Histórico de cirurgia cardíaca prévia e Acidente Vascular Cerebral foram definidas dicotomicamente em sim e não. A unidade de saúde que a família frequentava foi categorizada em ambulatorial UBS/USF, ambulatorial hospitalar ou centro de referência e hospital ou emergência. As informações sobre os pais foram coletadas como marcadores de risco adicional para os escolares.

Para a análise de dados foi criado um banco de dados no programa Excel for Windows e as análises conduzidas no pacote estatístico Stata (V.10.0), onde foram realizadas correções e eliminações de inconsistências. Foi realizada uma análise descritiva com o objetivo de identificar as características gerais e específicas da população estudada. Os resultados foram apresentados em tabelas e figura. $O$ projeto foi aprovado pela Plataforma Brasil/ CEP UNEB (Parecer 241.434/2013) e financiado pelo PRO Saúde/PET-Saúde 2012. Para participar do estudo os escolares manifestaram sua aquiescência por meio do Termo de Assentimento do Menor e seus pais sinalizaram concordância com a assinatura do Termo de Consentimento Livre e Esclarecido.

\section{RESULTADOS}

Foram entrevistados 43 dos 46 escolares que participaram do Programa "Doutores Mirins" na referida escola estadual. Dentre estes, 4 (9,3\%) foram excluídos por não estarem presentes nos dias da aplicação do instrumento da pesquisa, 1 $(2,3 \%)$ por recusar a finalizar $\circ$ instrumento de 
coleta e $1(2,3 \%)$ por ter deixado de frequentar a escola. Deste modo, a população do estudo foi composta com 37 escolares. Na Tabela 1 encontram-se as características sociodemográficas dos referidos escolares e de seus pais. Observouse que os escolares eram, predominantemente, do sexo feminino $(65,8 \%)$, com idades variando de 12 a 14 anos $(62,2 \%)$, e cor da pele preta ou parda $(91,9 \%)$ Em relação aos pais verificou-se que eram, majoritariamente, trabalhadores do setor de serviço ou autônomos (pais) $(83,8 \%$ ), assim como as mães $(67,6 \%)$ e pais e mães estudaram mais que 8 anos. Quanto à renda familiar foi detectado que $54,1 \%$ recebiam mais que 1 salário mínimo mensal.

Tabela 1. Características sociodemográficas de escolares egressos de um programa de educação em saúde e de seus pais. Salvador, Bahia, 2015. $n=37$

\begin{tabular}{|c|c|c|}
\hline Características sociodemográficas dos escolares & n & $\%$ \\
\hline \multicolumn{3}{|l|}{ Sexo } \\
\hline Masculino & 16 & 43,2 \\
\hline Feminino & 21 & 56,8 \\
\hline \multicolumn{3}{|l|}{ Idade (anos) } \\
\hline $10-11$ & 14 & 37,8 \\
\hline $12-14$ & 23 & 62,2 \\
\hline \multicolumn{3}{|l|}{ Cor da pele } \\
\hline Branca & 3 & 8,1 \\
\hline Preta/parda & 34 & 91,9 \\
\hline Características sociodemográficas dos pais & $\mathbf{n}$ & $\%$ \\
\hline \multicolumn{3}{|l|}{ Ocupação do paia } \\
\hline Setor de serviços/autônomo & 31 & 83,8 \\
\hline Desempregado & 6 & 16,2 \\
\hline \multicolumn{3}{|l|}{ Ocupação da mãe } \\
\hline Setor de serviços/autônoma & 25 & 67,6 \\
\hline Desempregada & 12 & 32,4 \\
\hline \multicolumn{3}{|l|}{ Escolaridade do pai (anos)a } \\
\hline$\leq 8$ & 13 & 37,1 \\
\hline$>8$ & 22 & 62,9 \\
\hline \multicolumn{3}{|l|}{ Escolaridade da mãe (anos)a } \\
\hline$\leq 8$ & 13 & 36,1 \\
\hline$>8$ & 23 & 63,9 \\
\hline \multicolumn{3}{|l|}{ Renda familiar mensal (em salários mínimos) } \\
\hline$\leq 1$ & 17 & 45,9 \\
\hline$>1$ & 20 & 54,1 \\
\hline
\end{tabular}

Na Tabela 2 estão dispostas as características clínicas e de hábitos de vida dos escolares. Constatou-se que $32,4 \%$ dos alunos apresentavam sobrepeso e $10,8 \%$ pressão arterial elevada. Com relação ao padrão de atividade física, observou-se que $24,3 \%$ foram considerados como insuficientemente ativos e $54,1 \%$ relataram tempo de tela superior a 2 horas diárias. Observou-se ainda que $21,6 \%$ já haviam experimentado bebidas alcoólicas. 
As variáveis relativas à história de saúde dos pais são apresentadas na Tabela 3. Os escolares relataram que pai ou mãe apresentavam HAS $(64,9 \%)$, e os mesmos referiram que $24,3 \%$ deles não cuidavam da HAS adequadamente. Citaram ainda história de Acidente Vascular Cerebral (18,9\%), de cirurgia cardíaca de $8,1 \%$ e busca por cuidados de saúde em hospitais ou emergências $(41,7 \%)$.
O padrão alimentar descrito pelos escolares encontrase exposto na Figura 1. Identificou-se frequência inadequada do consumo tanto de alimentos considerados como protetores, tais como saladas cruas $(78,4 \%)$, legumes e verduras cozidas $(94,6 \%)$ e frutas frescas $(81,1 \%)$, quanto para aqueles considerados como de risco, como salgadinhos de pacote $(72,9 \%)$ e biscoitos doces ou recheados $(72,9 \%)$.

Tabela 2. Características clínicas e hábitos de vida de escolares egressos do programa de educação em saúde. Salvador, Bahia, 2015 . $n=37$

\begin{tabular}{|c|c|c|}
\hline Características clínicas & n & $\%$ \\
\hline \multicolumn{3}{|l|}{ Índice de Massa Corpórea } \\
\hline Eutrófico & 25 & 67,6 \\
\hline Sobrepeso & 12 & 32,4 \\
\hline \multicolumn{3}{|l|}{ Pressc̃o Arterial } \\
\hline Normal & 33 & 89,2 \\
\hline Elevada & 4 & 10,8 \\
\hline \multicolumn{3}{|c|}{ Já experimentou bebidas alcoólicas } \\
\hline Sim & 8 & 21,6 \\
\hline Não & 29 & 78,4 \\
\hline \multicolumn{3}{|c|}{ Idade com que experimentou bebidas alcoólicasa } \\
\hline $10-11$ & 2 & 33,3 \\
\hline $12-14$ & 3 & 50,0 \\
\hline \multicolumn{3}{|l|}{ Atividade física ${ }^{1}$} \\
\hline Suficientemente ativo & 28 & 75,7 \\
\hline Insuficientemente ativo & 9 & 24,3 \\
\hline \multicolumn{3}{|c|}{ Tempo de tela ${ }^{2}$ (em horas por dia) } \\
\hline$<2$ & 17 & 45,9 \\
\hline$\geq 2$ & 20 & 54,1 \\
\hline
\end{tabular}

Tabela 3. História familiar de saúde de escolares egressos do programa de educação em saúde. Salvador, Bahia, 2015. n=37

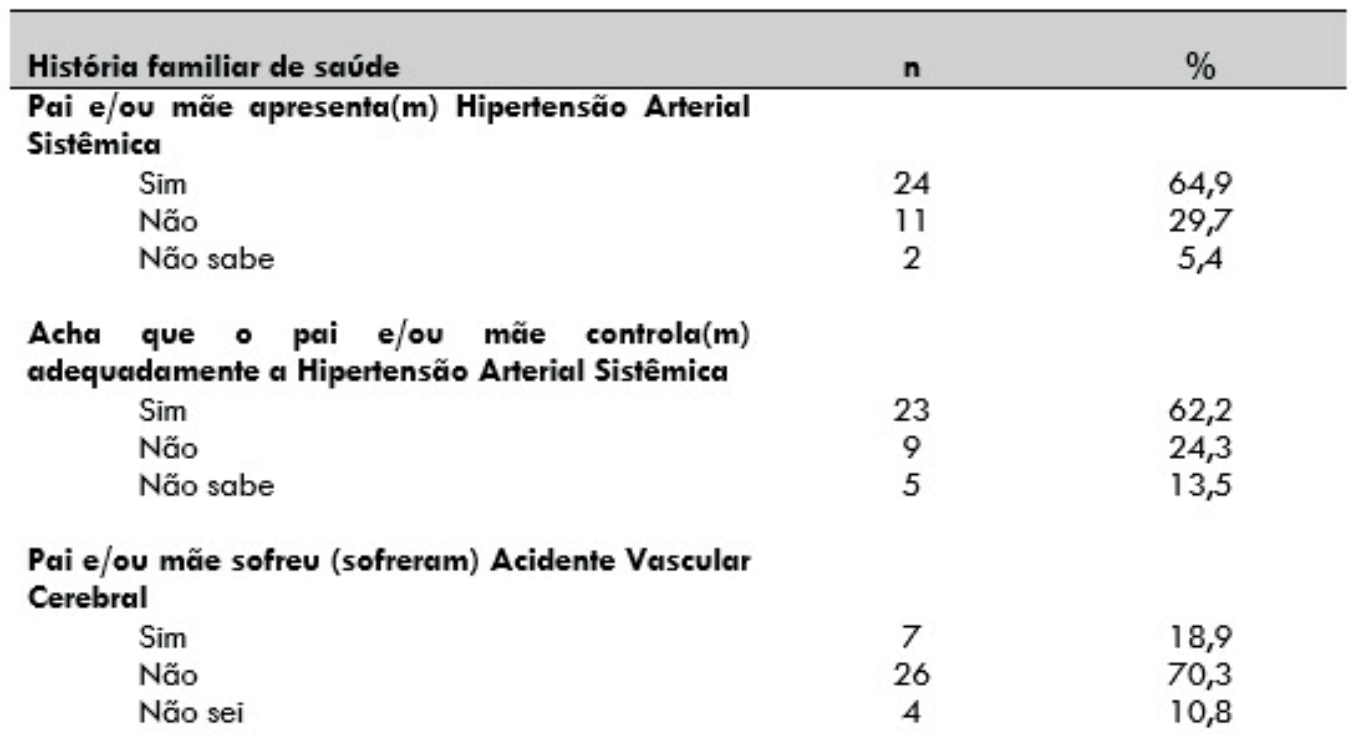


Pai e/ou mãe já fez (fizeram) cirurgia cardíaca

Sim

Não

Não sei

Unidade de saúde que a família frequenta

Ambulatorial (UBS/USF)

Ambulatorial (Hospitalar/Centro de Referência)

Hospital / Emergência

$\begin{array}{cc}3 & 8,1 \\ 28 & 75,7 \\ 6 & 16,2\end{array}$

10

27,7

30,6

41,7

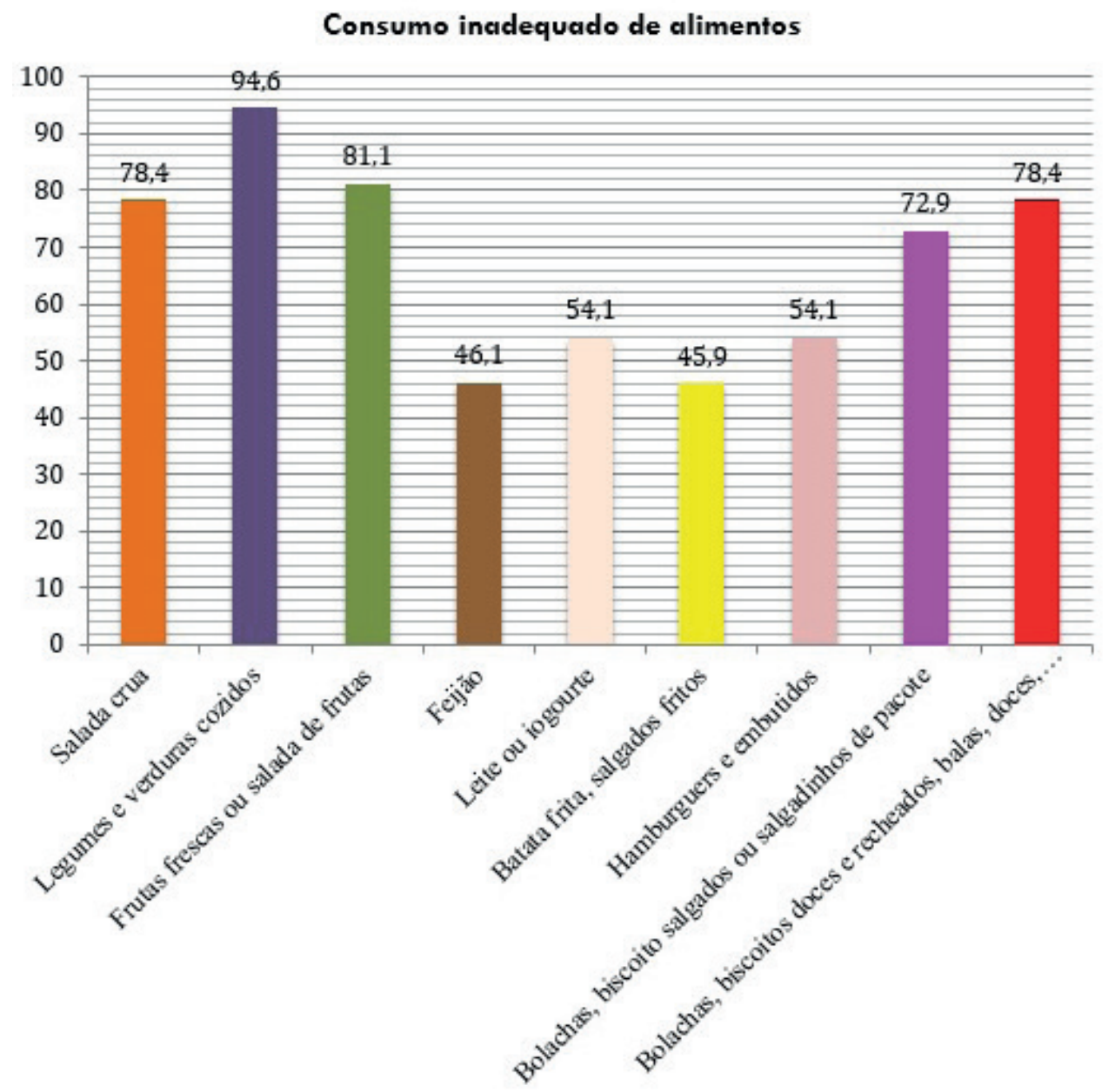

Figura 1. Consumo inadequado de alimentos por escolares egressos de programa de educação em saúde, Salvador, Bahia, $2015 . \mathrm{n}=37$.

\section{DISCUSSÃO}

A frequência de fatores de risco cardiovascular em escolares egressos de um programa multiprofissional de educação em saúde destinado a adoção de hábitos de vida saudável mostrou-se elevada.

A alta prevalência de doenças cardiovasculares em todo mundo, em adultos e idosos, já é bem documentada, todavia pouca atenção tem sido dirigida para a população mais jovem, constituída por crianças e adolescentes que está sujeita, como os mais velhos, a uma rotina de cada vez maior de inatividade física ${ }^{3,11,13-15}$, maior consumo de 
açúcares simples s,13-18, lipídios $^{5,13-18}$ e álcool ${ }^{12,14,16}$. A frequência de pressão arterial elevada relatada no presente estudo foi de $10,8 \%$, o que reafirma a necessidade de investimentos em educação em saúde para que hábitos de vida inadequados, como inatividade física, dieta hipercalórica e ingesta de bebidas alcoólicas, possam, gradativamente ser substituídos por um estilo de vida mais saudável ${ }^{5,19}$.

Níveis pressóricos elevados em escolares de escolas públicas foram também reportados por outros autores. Moura et al. ${ }^{20}$, estimaram prevalência de $13,7 \%$ de HAS em estudo realizado em escolas da região nordeste com uma amostra de 211 adolescentes de 12 a 18 anos. Farias Júnior et al. ${ }^{18}$, estimaram $18,0 \%$ de pressão elevada para adolescentes de 14 a 17 anos no Piauí. Evidências científicas de fatores de risco ambientais ${ }^{17,18,20,21}$ e genéticos ${ }^{2,22}$ já foram produzidas e encontram-se bem documentadas. No presente estudo, investigouse também a frequencia de HAS nos pais. De fato, um dos critérios de inclusão no programa Doutores Mirins foi a história familiar de HAS. Vários genes e polimorfismos têm sido estudados para a investigação da hipertensão hereditária, mas as mutações genéticas não estão ainda suficientemente claras a ponto de auxiliar no manejo da hipertensão ${ }^{2}$.

Frequências elevadas de sobrepeso, como encontradas no atual estudo, tem sido uma discussão constante entre pesquisadores, planejadores da área da saúde e de organizações não governamentais em função da magnitude do problema ${ }^{7,14-18,22}$.

A obesidade na infância e na adolescência foi documentada por vários autores que sinalizaram - aumento da prevalência nas últimas décadas em decorrência dos maus hábitos alimentares e da falta de atividade física ${ }^{4,14-18}$.

As evidências científicas reiteram que o sedentarismo de crianças e adolescentes é um fator de risco cardiovascular muito frequente ${ }^{3,4,11,14,19-21}$. Campos et al. $^{17}$ identificaram $22,6 \%$ das meninas foram consideradas como sedentárias e Lancarotti et al. $^{14}$, encontraram frequências de negação à prática de esportes ou competições variando de $14,4 \%$ a $32,1 \%$, majoritariamente para alunos do sexo feminino vinculadas a escolas públicas ${ }^{14}$. O sedentarismo pode ser justificado, não só pela utilização excessiva de jogos eletrônicos, considerados como um lazer passivo ${ }^{11,14,18,21}$, quanto pela indisponibilidade de uso de amplos espaços. O aumento da violência urbana tem sido reportado como um dos fatores que dificultam o acesso às ruas e consequentemente, a prática de brincadeiras de ruas ou esportes ${ }^{18}$. Brito et al. ${ }^{19}$, reiteraram ainda - papel das escolas na redução do sedentarismo ao estimular a prática de educação física e, desta forma, integrar ações de educação em saúde.

O elevado tempo desprendido com equipamentos eletrônicos, também chamado de tempo de tela ${ }^{11}$, foi um dos achados encontrados neste trabalho. Tais dados são semelhantes aos produzidos por outros autores, como Lancarotte et al. $^{14}$, que identificaram que de $56,0 \%$ a $70,5 \%$ dos adolescentes ficaram mais de duas horas à frente de TV, videogame ou computadores. As informações sistematizadas por pesquisadores responsáveis pela Pesquisa Nacional de Saúde Escolar (PeNSE) ${ }^{21}$, com escolares de 10 a 14 anos, sugerem que $79,5 \%$ também gastam mais de duas horas diárias em frente à TV. Reiterase que o tempo elevado destinado a atividades passivas é prejudicial também pela exposição a anúncios publicitários de alimentos industrializados, como sinalizado por Mattos et $\mathrm{al}^{24}$. Estes autores sugerem que a mídia televisiva estimula o consumo de alimentos considerados como de risco. Tais atividades, sedentarismo e consumo inadequado de alimentos processados, potencializam o risco de sobrepeso. Vale salientar também que, em função da já referida violência urbana, os pais podem ser incitados a comprar cada vez mais jogos eletrônicos e aparatos tecnológicos para manter seus filhos protegidos dentro de casa.

Assim como no presente estudo, o padrão alimentar desfavorável de escolares já foi detectado em outras pesquisas ${ }^{14,16,23,24}$. No presente estudo identificou-se frequência elevada de consumo de alimentos processados, e reduzida de saladas cruas, frutas frescas, legumes e verduras cozidas. Tais resultados corroboram os de Lancarotte et al. ${ }^{14}$, Pinto et al. ${ }^{16}$, e Tornquist et al. ${ }^{23}$. A alimentação é um dos aspectos que tem despertado a atenção dos profissionais da saúde em função dos riscos a longo prazo. $O$ consumo excessivo de doces, salgadinhos, refrigerantes, fastfoods, produtos enlatados e comidas gordurosas, fazem parte do cardápio dos escolares ${ }^{14,24}$. Uma das possíveis explicações seria - grande acesso a alimentos industrializados que 
cresceu muito nas últimas décadas ${ }^{14,24}$. Como eles são mais baratos e podem ser estocados, acabam sendo as opções mais frequentes para os pais que trabalham e não tem tempo para preparar as refeições. Estima-se que a baixa renda e o nível de escolaridade baixo exerça uma enorme influência na escolha de tais alimentos. Com 0 poder de compra reduzido, os alimentos mais processados são os mais acessíveis. Tais comportamentos podem ser reforçados ainda pela falta de esclarecimento sobre seus malefícios contribuindo para que sejam cada vez mais comprados.

Com relação ao consumo de bebidas alcoólicas, salienta-se que a OMS também o classifica como fator de risco para o desenvolvimento de doenças do aparelho circulatório e propõe sua redução no contexto de uma abordagem integrada para todas as faixas etárias'. Pesquisadores apontam que o álcool é uma variável que sinaliza adições e se encontra, muito frequentemente, associada ao aumento do consumo de lipídios e menor atividade física concorrendo para a HAS ${ }^{12,21}$. No presente estudo, os resultados corroboram os de outros pesquisadores. Malta et al. ${ }^{12}$ e Malta et al. ${ }^{21}$ relataram que $50,3 \%$ de escolares brasileiros de 13 a 15 anos já haviam experimentado bebidas alcoólicas. Na PeNSE ${ }^{21}$, com dados representativos para o país, foi estimado que o consumo da primeira dose ocorreu entre as idades de 12 e 13 anos. Uma das possíveis explicações para tais fatos seria a elevada frequência de consumo de álcool entre os adultos e o convívio com esta substância no próprio domicílio. Vale salientar que o álcool constitui um veículo para inserção social, principalmente por adolescentes necessitam de aceitação nesta fase da vida ${ }^{21}$. Este fato contribui para que, dentre as drogas lícitas, ele seja o mais utilizado entre crianças e adolescentes e tão precocemente. Os familiares e os amigos são, muitas vezes, os responsáveis por estimularem a experimentação do álcool e seu consumo entre crianças e adolescentes.

Com relação ao perfil de saúde dos pais, chama a atenção a elevada prevalência de HAS e - uso de hospitais e emergências para cuidar da saúde. Tais aspectos podem sugerir baixa compreensão acerca da cronicidade da doença e não aderência ao tratamento medicamentoso. Esses fatores são, muitas vezes, os principais motivos para a procura por serviços de emergência ${ }^{25}$. É também um comportamento considerado como de risco para o surgimento de crises hipertensivas e $\mathrm{AVC}^{25}$. Salienta-se ainda que tais práticas podem ser prejudiciais aos adolescentes em termos de educação em saúde. Perceber que seus pais buscam frequentemente cuidados em emergências pode suscitar o entendimento desta prática como correta, desvalorizando a oferta de cuidados em unidades básicas de saúde, que deveria ser sempre a porta de entrada no Serviço Único de Saúde.

É interessante ressaltar algumas fragilidades do estudo. O emprego do desenho transversal impossibilita inferências causais, muito embora tal metodologia tenha relevância para caracterizar a população e levantar possíveis fatores de risco. O número amostral reduzido também pode ser apontado como uma fragilidade já que não possibilita a realização de análises mais apropriadas e consequente extrapolação de resultados para escolares desta faixa etária em geral. A medida da pressão arterial realizada em um único dia, mesmo em duas medições, pode também ser apontado como limitação.

\section{CONCLUSÃO}

Os resultados permitem concluir que a frequência de fatores de risco cardiovascular se mostrou elevada para os escolares de 10 a 14 anos. Pressão arterial alterada, sedentarismo, sobrepeso/ obesidade, dieta inadequada e contato precoce com bebidas alcoólicas são achados importantes para os adolescentes, que devem suscitar reflexões e ações. A prevalência elevada de hipertensão, histórico de cirurgia cardíaca, AVC e eleição de hospitais e emergências para cuidar da saúde entre os pais, também são fatores preocupantes que podem sinalizar baixa adesão ao tratamento medicamentoso e necessidade de educação em saúde para a família. Diante deste contexto, recomenda-se a aferição rotineira da pressão arterial de escolares, a implantação de estratégias para controle dos fatores de risco ambientais na atenção primária, assim como ênfase em grupos de educação em saúde que podem empoderar indivíduos hipertensos, de qualquer faixa etária, e 
auxiliá-los no cuidado global à saúde.

\section{REFERÊNCIAS}

1. World Health Organization (WHO). Cardiovascular Diseases [Internet]. 2015 [Acesso em Jan 2015]. Disponível em: http://www.who.int/ mediacentre/factsheets/fs317/en/

2. Sarkar T, Singh NP. Epidemiology and Genetics of hypertension. Journal of the Association of Physicians of India. 2015;63:61-67.

3. Corrêa Neto VG, Sperandei S, Silva LAI, Maranhão Neto GA, Palma A. Hipertensão arterial em adolescentes do Rio de Janeiro: prevalência e associação com atividade física e obesidade. Ciência \& Saúde Coletiva. 2014;19(6):1699-1708. doi: $10.1590 / 1413-81232014196.05262013$

4. Quadros FF, Gutierrez LLP, Ribeiro JL. Obesidade e fatores de risco cardiovascular em filhos de obesos: uma revisão. Scientia Medica. 2013;23(2): $119-126$.

5. Costa JV, Silva ARV, Moura IH, Carvalho RBN, Bernardes LE, Almeida PC. Análise dos fatores de risco para hipertensão arterial em adolescentes escolares. Rev. Latino-Am. Enfermagem. $2012 ; 20(2)$ : 1-7. doi: 10.1590/S010411692012000200011

6. Carmo Júnior TR. A intervenção escolar como meio de promover o estilo de vida ativo em escolares de 7 a 11 anos. [Dissertação - Mestrado em Educação Física]. Brasília: Universidade Católica de Brasília; 2009.

7. Cole TJ, Bellizzi MC, Flegal KM, Dietz WH, Cole TJ, Bellizzi MC, et al. Estabilishing a standard definition for child overweight and obesity worldwide: international survey. 2000;6(320):1-6. doi: $10.1136 /$ bmj.320.7244.1240

8. VI Diretrizes Brasileiras de Hipertensão. Arq Bras Cardiol. 2010;Supl1:1-40.

9. Brasil. Ministério da Saúde. Secretaria de atenção à Saúde. Departamento de atenção básica. Uso dos formulários e registros das informações no Sisvan web. Brasília. DF.

10. Farias Júnior JC, Lopes AS, Mota J, Santos MP, Ribeiro JC, Hallal PC. Validade e reprodutibilidade de um questionário para medida de atividade física em adolescentes: uma adaptação do SelfAdministered Physical Activity Checklist. Rev Bras Epidemiol. 2012;15(1):198-210. doi: 10.1590/ S1415-790X2012000100018

11. Friedrich RR, Polet JP, Schuch I, Wagner MB. Effect of intervention programs in schools to reduce screen time: a meta-analysis. J Pediatr (Rio J). 2014;90(3):232-241. doi: 10.1016/i. iped.2014.01.003

12. Malta DC, Machado IE, Porto DL, MMA Silva, Freitas PC, Costa AWN et al. Consumo de álcool entre adolescentes brasileiros segundo a Pesquisa Nacional de Saúde Escolar (PeNSE 2012). Rev Bras Epidemiol Suppl PeNSE 2014;203-214. doi: $10.1590 / 1809-4503201400050016$

13. Ribas SA, Silva LCS. Fatores de risco cardiovascular e fatores associados em escolares do Município de Belém, Pará, Brasil. Cad. Saúde Pública. 2014;30(3):577-586. doi: $10.1590 / 0102-311 \times 00129812$

14. Lancarotte I, Nobre MR, Zanetta R, Polydoro $M$. Lifestyle and cardiovascular health in school adolescents from São Paulo. Arq. Bras. Cardiol. 2010;95(1):61-69. doi: 10.1590/S0066$782 \times 2010005000077$

15. Guerra PH, Farias Junior JC, Florindo AA. Comportamento sedentário em crianças e adolescentes brasileiros: revisão sistemática. Rev. Saúde Pública. 2016;50(9):1 -15. doi: 10.1590/ S1518-8787.2016050006307

16. Pinto SL, Silva RCR, Priore SE, Assis AMO, Pinto EJ. Prevalência de pré-hipertensão e hipertensão e avaliação de fatores associados em crianças e adolescentes de escolas públicas de Salvador, Bahia, Brasil. Cad. Saúde Pública. 2011 ;27(6):1065-1076. doi: 10.1590/S0102$311 \times 2011000600004$ 
17. Campos W, Stabelini Neto A, Bozza R, Ulbrich AZ, Bertin RL, Mascarenhas LPG et al. Atividade física, consumo de lipídios e fatores de risco para aterosclerose em adolescentes. Arq Bras Cardiol. 2010;94(5):601-7. doi: 10.1590/S0066$782 \times 2010005000033$

18. Farias Júnior JC de, Mendes JKF, Barbosa DBM, Lopes A da S. Fatores de risco cardiovascular em adolescentes : prevalência e associação com fatores sociodemográficos. Rev Bras Epidemiol. 2011 ; 14(1):50-62. doi: 10.1590/S1415$790 \times 2011000100005$

19. Brito AKA, Silva FIC, França NA. Programas de intervenção nas escolas brasileiras: uma contribuição das escolas para a educação em saúde. Saúde em Debate. 201 2;36(95):624-632. doi: $10.1590 /$ S0103-11 1042012000400014

20. Moura IH, Vieira EES, Silva GRF, Carvalho RBN, Silva ARV. Prevalência de hipertensão arterial e seus fatores de risco em adolescentes. Acta Paul Enferm. 2015;28(1):81-6. doi: 10.1590/19820194201500014

21. Malta DC, Sardinha LMV, Mendes I, Barreto SM, Giatti L, Castro IRRC et al. Prevalência de fatores de risco e proteção de doenças crônicas não transmissíveis em adolescentes: resultados da Pesquisa Nacional de Saúde do Escolar (PeNSE), Brasil, 2009. Ciênc. Saúde Coletiva. 2010;15(Suppl2):3009-3019. doi: 10.1590/ S1413-81232010000800002

22. Mazor-Aronovitch K, Lotan D, Modan-Moses $D$, Fradkin A, Pinhas-Hamiel O. Blood pressure in obese and overweight children and adolescents. Isr Med Assoc J. 2014;16(3):157-61.

23. Tornquist $L$, Tornquist $D$, Reuter $C P$, Burgos LT, Burgos MS. Excesso de peso e pressão arterial elevada em escolares: prevalência e fatores associados. Journal Human Growth and Development. 2015;25(2):216-223. doi: 10.7322/ jhgd.103018

\section{Mattos MC, Nascimento PCBD, Almeida}

SS, Costa TMB. Influência de propaganda de alimentos nas escolhas alimentares de crianças e adolescentes. Psicologia: Teoria e Prática.
$2010 ; 12(3): 34-51$.

25. Vancini-Campanharo CR, Oliveira GN, Andrade TFL, Okuno MFP, Lopes MCBT, Batista REA. Hipertensão Arterial Sistêmica no Serviço de Emergência: adesão medicamentosa e conhecimento da doença. Rev. Latino-Am Enfermagem. 2015;23(6):1 149-56. doi: 10.1590/0104-1169.0513.2660 